Western University Scholarship@Western

$1-29-2017$

\title{
Sleep Spindles and Intellectual Ability: Epiphenomenon or Directly Related?
}

Zhuo Fang

Western University

Valya Sergeeva

Western University

Laura B. Ray

Western University

Adrian M. Owen

Western University, dpavich@uwo.ca

Stuart M. Fogel

Western University; University of Ottawa, sfogel@uwo.ca

Follow this and additional works at: https://ir.lib.uwo.ca/anatomypub

Part of the Anatomy Commons, and the Cell and Developmental Biology Commons

Citation of this paper:

Fang, Zhuo; Sergeeva, Valya; Ray, Laura B.; Owen, Adrian M.; and Fogel, Stuart M., "Sleep Spindles and Intellectual Ability: Epiphenomenon or Directly Related?" (2017). Anatomy and Cell Biology Publications. 73.

https://ir.lib.uwo.ca/anatomypub/73 


\title{
Sleep Spindles and Intellectual Ability: Epiphenomenon or Directly Related?
}

\author{
Zhuo Fang ${ }^{1}$, Valya Sergeeva ${ }^{1}$, Laura B. Ray ${ }^{1}$, Jeremy Viczko ${ }^{1}$, \\ Adrian M. Owen ${ }^{1}$, and Stuart M. Fogel ${ }^{1,2}$
}

\begin{abstract}
Sleep spindles - short, phasic, oscillatory bursts of activity that characterize non-rapid eye movement sleep-are one of the only electrophysiological oscillations identified as a biological marker of human intelligence (e.g., cognitive abilities commonly assessed using intelligence quotient tests). However, spindles are also important for sleep maintenance and are modulated by circadian factors. Thus, the possibility remains that the relationship between spindles and intelligence quotient may be an epiphenomenon of a putative relationship between good quality sleep and cognitive ability or perhaps modulated by circadian factors such as morningness-eveningness tendencies. We sought to ascertain whether spindles are directly or indirectly related to cognitive abilities using mediation analysis. Here, we show that fast $(13.5-16 \mathrm{~Hz})$ parietal but not slow $(11-13.5 \mathrm{~Hz})$ frontal spindles
\end{abstract}

\section{INTRODUCTION}

One of the only neural oscillations identified as a biological marker of human intelligence (cognitive abilities commonly assessed using intelligence quotient [IQ] tests) is the sleep spindle (for a review, see Fogel \& Smith, 2011). The investigation of the link between spindles and human intelligence can serve as a window to understanding the neural basis of cognitive abilities that support, for example, the capacity for reasoning (i.e., to identify complex patterns and relationships and the use of logic) and existing knowledge, skills, and experience that can be used to solve novel problems. Sleep spindles are neural oscillations ( $11-16 \mathrm{~Hz}$; Iber, Ancoli-Israel, Chesson, \& Quan, 2007) that occur in short bursts ( $<3 \mathrm{sec})$ and characterize non-rapid eye movement stage 2 (NREM2) sleep. Within an individual, the characteristics of sleep spindles are very trait-like and are stable from night to night but vary significantly between individuals (Gaillard \& Blois, 1981; Silverstein \& Levy, 1976) and have thus been suggested to be an "electrophysiological fingerprint" for each individual (De Gennaro, Ferrara, Vecchio, Curcio, $\&$ Bertini, 2005). However, the functional significance of this fingerprint remains to be fully elucidated. Studies in${ }^{1}$ Western University, London, Ontario, Canada, ${ }^{2}$ University of
Ottawa, Ontario, Canada in both non-rapid eye movement stage 2 sleep and slow wave sleep are directly related to reasoning abilities (i.e., cognitive abilities that support "fluid intelligence," such as the capacity to identify complex patterns and relationships and the use of logic to solve novel problems) but not verbal abilities (i.e., cognitive abilities that support "crystalized intelligence"; accumulated knowledge and experience) or cognitive abilities that support STM (i.e., the capacity to briefly maintain information in an available state). The relationship between fast spindles and reasoning abilities is independent of the indicators of sleep maintenance and circadian chronotype, thus suggesting that spindles are indeed a biological marker of cognitive abilities and can serve as a window to further explore the physiological and biological substrates that give rise to human intelligence. vestigating the functions of sleep spindles have identified three main roles: (1) for memory consolidation, (2) in relation to intellectual abilities, and (3) for sleep maintenance and protection.

Sleep spindles have been shown to relate to memory, primarily in terms of learning-dependent, night-to-night variations in sleep spindle characteristics (e.g., density, amplitude, duration). More specifically, NREM2 sleep enhances the consolidation of both declarative (e.g., Fogel \& Smith, 2006; Clemens, Fabó, \& Halász, 2005; Schabus et al., 2004; Gais, Mölle, Helms, \& Born, 2002) and procedural (e.g., Barakat et al., 2011; Morin et al., 2008; Fogel, Nader, Cote, \& Smith, 2007; Fogel \& Smith, 2006; Nader \& Smith, 2003; Fogel \& Jacob, 2001, 2002) memory after new learning via the action of sleep spindles (for a review, see Rasch \& Born, 2013; Fogel \& Smith, 2011).

Sleep spindles also have been shown to closely relate to cognitive abilities, primarily in terms of rather stable interindividual differences in cognitive abilities, typically assessed by IQ tests. For example, Nader and Smith (Fogel \& Smith, 2006; Nader \& Smith, 2001, 2003) identified a correlation in both the number of sleep spindles and sigma power $(12-14 \mathrm{~Hz})$ with performance IQ as measured by the Multidimensional Aptitude Battery-II (MAB-II IQ; Jackson, 1998). On the other hand, higher spindle density has also been found to predict better performance on 
neuropsychological assessments in elderly participants, aged 50-91 years, including measures of verbal learning, visual attention, and verbal fluency (Lafortune et al., 2014). Given that verbal and performance-related abilities are highly intercorrelated, to explore whether performance or verbal IQ-related abilities were uniquely correlated with spindles, Fogel et al. (2007) controlled for the individual differences in verbal IQ and identified that sleep spindles and sigma power were uniquely correlated with performance IQ over and above verbal IQ, thus suggesting that sleep spindles may be specifically related to performanceIQ-related abilities (i.e., "fluid intelligence"; cognitive abilities that support the capacity for reasoning) as opposed to verbal-IQ-related abilities (i.e., "crystalized intelligence"; the use of existing knowledge, skills, and experience using words and numbers). Consistent with these findings, spindle density was found to be correlated with performance on the Raven's Standard Progressive Matrices (Bódizs et al., 2005), an intelligence test that measures reasoning ability (Raven, Court, \& Raven, 1976). Furthermore, Bódizs, Lázár, and Rigó (2008) identified a significant positive correlation between right-parietal, fast sleep spindle density and visuospatial abilities, measured by the Rey-Osterrieth Complex Figure test. Most recently, in a large sample of participants, Ujma and colleagues (2014) identified a positive correlation between intelligence (as measured by the Cattell Culture Fair Intelligence Test and the Standard Progressive Matrices) and fast spindle amplitude in central and frontal derivations and also with slow spindle duration across most areas of the scalp in women, but not in men, thus suggesting that the relationship between spindles and IQ may be stronger in women than in men. Taken together, these studies support the notion that sleep spindles are electrophysiological markers of cognitive abilities, in particular, the ability to reason and solve new problems using logic and reasoning. However, up until now, this research has not considered whether the relationship between spindles and cognitive ability might be explained (or mediated) by other related factors. More specifically, related functions that spindles support such as sleep quality or factors that modulate spindles and relate to cognitive performance, such as circadian rhythmicity, may partially (or entirely) explain this relationship; however, this possibility remains to be explicitly tested.

Sleep spindles have also been shown to protect sleep from external stimuli (Schabus et al., 2012; Dang-Vu, McKinney, Buxton, Solet, \& Ellenbogen, 2010; Vyazovskiy, Achermann, Borbely, \& Tobler, 2004; Cote, Epps, \& Campbell, 2000; Elton et al., 1997; Peters \& Jones, 1991). For example, Dang-Vu and colleagues (2010) demonstrated that individuals who generate a greater number of sleep spindles maintain sleep better in the face of disruptive stimuli. In addition, brain responses to external auditory stimuli are absent when the stimuli are presented during a sleep spindle (Schabus et al., 2012; Cote et al., 2000), but not outside spindle events. Furthermore, related studies also demonstrated that sleep spindles measured on a normal, quiet/undisturbed night of sleep were predictive of sleep quality on a subsequent night in a noisy environment (Dang-Vu et al., 2010). Moreover, baseline sleep spindles during a normal, quiet/undisturbed night of sleep could predict scores on the insomnia severity index during a disturbed night of sleep (Dang-Vu et al., 2015). In other words, sleep spindles from a normal, undisturbed night of sleep have predictive value (and are thus valid measures) of the continuity of sleep. On the basis of this, we would predict that spindles measured on a normal, natural night of sleep would be related to sleep quality. There is evidence to suggest that sleep quality is also related to cognitive abilities on tests that assess memory, attention, and executive functioning (Nebes, Buysse, Halligan, Houck, \& Monk, 2009; Bastien et al., 2003; Hauri, 1997; Bonnet \& Arand, 1995). More specifically, in elderly good sleepers and unmedicated patients with insomnia, those with higher objective sleep quality (from overnight polysomnography [PSG]) and subjective sleep quality (from sleep diaries) have been found to have enhanced intellectual performance on verbal and visual memory, attention, concentration, and executive functioning than those with lower sleep quality (Bastien et al., 2003). In older adults, poor sleep has been associated with lower global cognitive function and poorer performance on executive function and attention tests (Blackwell et al., 2006) as well as on tests measuring working memory, attentional set shifting, and abstract problem solving (Nebes et al., 2009). Thus, taken together, these studies suggest that sleep quality might relate to a wide range of cognitive abilities that depend on verbal abilities and STM and, perhaps, reasoning abilities. Thus, one of the aims of this study was to investigate whether sleep quality may partially explain or mediate the relationship between sleep spindles and cognitive abilities.

Sleep spindles are the only known NREM electrophysiological event modulated by the circadian pacemaker (Dijk \& Cajochen, 1997; Dijk \& Czeisler, 1995; Dijk et al., 1995; Dijk, Hayes, \& Czeisler, 1993). For example, in young participants, spindle amplitude reaches a minimum, and spindle frequency reaches its nadir around the trough of the body temperature cycle, whereas spindle duration and incidence reach a maximum around the nadir of the body temperature cycle (Wei, Riel, Czeisler, \& Dijk, 1999), suggesting that spindles are modulated by circadian influences. There is also evidence to suggest that circadian characteristics, such as circadian chronotype (e.g., morning, neutral, or evening types), are also related to cognitive abilities (Achilles, 2003; Song \& Stough, 2000; Roberts \& Kyllonen, 1999). However, evidence to support such a relationship is sparse, and results are less clear between studies. For example, Achilles (2003) identified that evening types have lower academic performance than morning types. However, another study (Roberts \& Kyllonen, 1999) involving United States Air Force recruits found that evening types scored higher on measures of memory and processing speed than morning types. On the other hand, 
Song and Stough (2000) identified no relationship between intellectual abilities, as measured by the MAB-II and the Inspection Time task, and chronotype. A metaanalysis (Preckel, Lipnevich, Schneider, \& Roberts, 2011) revealed a small but significant correlation between eveningness tendencies and cognitive ability $(r=.08)$ and with academic achievement $(r=-.14)$. Conversely, morningness tendencies demonstrated the opposite trend, with morningness relating to higher academic achievement $(r=.16)$ and lower cognitive abilities $(r=-.04$; Preckel et al., 2011). Further research is required to resolve existing inconsistencies in the literature about the relationship between cognitive ability and circadian chronotype. Although the relationship between circadian rhythmicity and sleep patterns (Daan, Beersma, \& Borbely, 1984; Borbely, 1982; for a review, see Schmidt, Collette, Cajochen, \& Peigneux, 2007) is well established, more recently, differences in sleep architecture have been reported between morning and evening types. Specifically, Mongrain, Carrier, and Dumont (2005) have reported that morning types but not evening types demonstrate higher EEG power in the low sigma (12-14 Hz) range during sleep, which further obfuscates the relationship between cognitive ability, sleep characteristics (such as spindles), and circadian factors (Mongrain et al., 2005). Thus, one of the aims of the current study is to investigate whether morningnesseveningness tendencies may partially explain the relationship between spindles and cognitive abilities.

Taken together, the extant literature supports the notion that sleep spindles are closely related to intellectual abilities, sleep maintenance, and circadian rhythmicity. Thus, the possibility remains that these interrelated factors may either partially or even entirely explain the relatively well-established relationship between spindles and cognitive abilities. The goal of the current investigation was to disentangle whether spindles are directly correlated with intellectual ability or if this represents some epiphenomenon of a link between better sleep quality or circadian influences and intelligence. Until now, investigations of the link between spindles and cognition have limited their focus to spindles alone, and to our knowledge, no studies have specifically tested whether other factors contribute to this relationship in the same set of participants. Thus, it remains unclear whether the relationship between sleep spindles and cognitive abilities is a direct effect or whether it is mediated by sleep quality or circadian rhythmicity. Here, we aim to explore the relationship between cognitive abilities and different features of sleep, including sleep spindles, commonly used measures of sleep quality, and morningness-eveningness tendencies to understand how directly related sleep spindles are to human intelligence. Specifically, in this study, we tested whether spindles were uniquely correlated with cognitive abilities (reasoning, verbal, and STM) and whether this relationship is either partially or fully mediated by either sleep quality or morningness-eveningness tendencies.

\section{METHODS \\ Participants}

Twenty-seven young, healthy adults (18 women; age = $26 \pm 6.69$ years, age range $=19-40$ years) from two related studies (study 1: $n=15$, study 2: $n=12$ ) were included in this study. Both studies adhered strictly to the same protocol, including all exclusion/inclusion criteria, timing of the data collection, materials and equipment, geographical location, and recruitment from the same population, which were all identical, thus precluding the possibility that pooling data from two separate studies would have any impact on the data or results. All participants reported normal sleep patterns (between $11 \mathrm{pm}$ and $9 \mathrm{am}$ ), were nonshift workers and medication-free, had no history of head injury or seizure, had a normal body mass index $(<25)$, and did not consume excessive nicotine or alcohol. To be included, interested participants had to score $<10$ on the Beck Depression Inventory (Beck, Rial, \& Rickels, 1974) and the Beck Anxiety Inventory (Beck, Epstein, Brown, \& Steer, 1988) and have no signs of sleep disorders indicated by the Sleep Disorders Questionnaire (Douglass et al., 1994). All participants underwent an initial night of PSG recording that served as an acclimatization/sleep disorder screening night. The screening night included EEG recordings (via electrodes applied to their scalp and face, including EEG channels Fz, Cz, Pz, and Oz), respiration (via thorax and abdomen belts), electrocardiographic activity (via electrodes placed below each clavicle), leg muscle activity (via electrodes placed on the outer, anterior tibialis muscle of each leg), and blood oxygen saturation (via a finder probe placed on the index finger of the right hand). Screening night recordings were scored in accordance with clinical scoring guidelines established by the American Academy of Sleep Medicine (Iber et al., 2007). Participants were excluded from further participation in the study if the results of their screening night identified greater than five respiratory events per hour of sleep or greater than 10 periodic leg movements per hour of sleep. Participants were also asked to wear an "Actiwatch" (Philips-Respironics, Inc., Andover, MA; a wrist-worn accelerometer to measure sleep-wake-related limb movements) and to complete a log of their daily activities and sleep habits, to verify that they maintained a regular sleep schedule for the length of their participation in the study. Participants were excluded from further participation in the study if the results of their Actiwatch or sleep diary identified variability in their sleep schedule outside the aforementioned exclusion criteria or noncompliance with the study protocol.

\section{Ethics Statement}

All participants were given a letter of information, provided informed written consent before participation, and were financially compensated for their participation. 
Table 1. Descriptive Statistics (Mean $\pm S D$ ) for Sleep Spindles and Sleep Architecture

\begin{tabular}{|c|c|c|c|c|c|c|c|c|}
\hline & & Stage & Amplitude & $p$ & Density & $p$ & Duration & $p$ \\
\hline \multirow[t]{5}{*}{ Sleep spindles } & Fast spindles & NREM2 & $33.17 \pm 8.16$ & .013 & $3.23 \pm 1.06$ & .009 & $0.65 \pm 0.08$ & $<.001$ \\
\hline & & SWS & $31.24 \pm 8.33$ & & $3.78 \pm 1.45$ & & $0.57 \pm 0.08$ & \\
\hline & Slow spindles & NREM2 & $35.11 \pm 8.54$ & .011 & $4.02 \pm 0.92$ & $<.001$ & $0.58 \pm 0.08$ & $<.001$ \\
\hline & & SWS & $32.96 \pm 9.13$ & & $5.66 \pm 1.29$ & & $0.52 \pm 0.07$ & \\
\hline & & & $S E$ & & $N A$ & & $M E Q$ & \\
\hline \multirow[t]{2}{*}{ Sleep architecture } & & & $92.83 \pm 5.03$ & & $17.00 \pm 7.10$ & & $52.93 \pm 9.67$ & \\
\hline & Wake & NREM1 & NREM2 & & SWS & & REM & \\
\hline Duration (min) & $34.03 \pm 25.38$ & $11.82 \pm 4.89$ & $191.91 \pm 44.60$ & & $127.53 \pm 37.89$ & & $323.32 \pm 24.83$ & \\
\hline$\%$ TST & $7.67 \pm 6.30$ & $2.78 \pm 1.12$ & $45.27 \pm 10.63$ & & $30.02 \pm 9.04$ & & $23.16 \pm 5.72$ & \\
\hline
\end{tabular}

NREM1 = NREM sleep stage 1; NREM2 = NREM sleep stage 2; SWS = slow wave sleep; SE = sleep efficiency; NA = number of awakenings; MEQ = Horne-Ostberg Morningness-Eveningness Questionnaire.

This research was approved by the Western University Health Science research ethics board.

\section{PSG Recording and Analysis}

Embla Titanium (Natus, San Carlos, CA) PSG systems were used to acquire in-laboratory PSG recordings. Physiological data were recorded at a sampling rate of $512 \mathrm{~Hz}$, with a high-pass filter of $0.15 \mathrm{~Hz}$ and low-pass filter of $220 \mathrm{~Hz}$. EEG, EOG, and EMG recordings were taken using gold-plated electrodes applied to the skin. The EEG (F3, $\mathrm{Fz}, \mathrm{F} 4, \mathrm{C} 3, \mathrm{Cz}, \mathrm{C} 4, \mathrm{P} 3, \mathrm{Pz}, \mathrm{P} 4$, and Oz) and EOG (from the left and right outer canthi of the eye) were recorded and rereferenced offline to the contralateral mastoid derivations (M1 and M2). The EMG (submental chin muscles) channel was recorded as a bipolar derivation. Sleep stages were scored in accordance with standard criteria (Iber et al., 2007) using RemLogic analysis software (Natus).

Automatic spindle detection was carried out using a previously published (Fogel, Ray, Binnie, \& Owen, 2015; Fogel et al., 2014; Albouy et al., 2013) and validated (Ray et al., 2015) in-house method employing EEGlab-compatible (Delorme \& Makeig, 2004) software (github.com/stuartfogel/ detect_spindles) written for MATLAB R2014a (The MathWorks Inc., Natick, MA). The detailed processing steps and procedures are reported elsewhere (Ray et al., 2015) and are thus presented only briefly here. The EEG data were initially downsampled to $128 \mathrm{~Hz}$. The detection was performed at $\mathrm{Fz}$ and $\mathrm{Pz}$ derivations. The spindle data were extracted from movement artifact-free, NREM sleep epochs (NREM2 and slow wave sleep [SWS] separately). The detection method (Ray et al., 2015) used a complex demodulation transformation of the EEG signal with a bandwidth of $5 \mathrm{~Hz}$ centered about a carrier frequency of $13.5 \mathrm{~Hz}$ (i.e., 11-16 Hz; Iber et al., 2007). Each data point was trans- formed into a $z$ score using the mean and standard deviation derived from a 60 -sec sliding window. Events (spindle onsets, peaks, and offsets) were then detected on the transformed signal with a $z$ score threshold of $z=$ 2.33. The dependent variables of interest extracted from this method include spindle amplitude, spindle duration, and spindle density (number of spindles per minute of NREM sleep) for each participant and at each derivation (Fz and $\mathrm{Pz}$ ). Despite having no minimum detection criteria, the detection method employed here did not detect spindles lower than $0.2 \mathrm{sec}$, as found in a previous validation study (Ray et al., 2015). Here, we confirmed that spindles less than $0.2 \mathrm{sec}$ were not included in the analyses. Spindles were categorized so that they were orthogonal at the scalp locations where they predominate topographically (Werth, Achermann, \& Borbely, 1997; Werth, Achermann, Dijk, \& Borbely, 1997; Zeitlhofer et al., 1997; Jobert, Poiseau, Jahnig, Schulz, \& Kubicki, 1992) as slow spindles (11-13.5 Hz) at Fz and fast spindles (13.5$16 \mathrm{~Hz}$ ) at $\mathrm{Pz}$ (Table 1).

\section{Intelligence Testing}

The Cambridge Brain Sciences (CBS) Trials platform (Hampshire, Highfield, Parkin, \& Owen, 2012) is a Webbased battery of cognitive tests that assess a broad range of cognitive abilities including reasoning, problem solving, planning, attention, and memory. The CBS Trials have a number of advantages over other tests of cognition, including ease of administration and the fact that the neural correlates of each subtest have been investigated using functional neuroimaging (Hampshire et al., 2012). A recent parcellation based on scores from 44,600 participants has revealed three factors that govern performance across the battery, which have been described as 
Table 2. Descriptive Statistics of the Three CBS Subscales (Reasoning, STM, and Verbal)

\begin{tabular}{lccc}
\hline IQ Measures & Range & Mean \pm SD & Median \\
\hline Reasoning & $83.9-114.17$ & $97.08 \pm 7.97$ & 94.68 \\
STM & $93.51-135.81$ & $103.01 \pm 9.36$ & 101 \\
Verbal & $90.80-121.93$ & $101.22 \pm 6.93$ & 100.37 \\
\hline
\end{tabular}

"reasoning ability," "verbal ability," and "STM" (Hampshire et al., 2012). The Reasoning factor is best described in terms of performance on five tests adapted from the cognitive literature, including deductive reasoning (Boyle, 1988; Cattell, 1949), spatial rotation (Silverman et al., 2000), feature match (Treisman \& Gelade, 1980), spatial planning (Shallice, 1982), and interlocking polygons (Folstein, Folstein, \& McHugh, 1975). STM can be best described in terms of four tests, including visuospatial working memory (Inoue \& Matsuzawa, 2007), spatial span (Corsi, 1972), paired associates (Gould, Brown, Owen, Bullmore, \& Howard, 2006), and self-ordered search (Collins, Roberts, Dias, Everitt, \& Robbins, 1998). Finally, Verbal ability is best captured by performance on three tests, including verbal reasoning (Baddeley, 1968), color-word remapping (Stroop, 1935), and digit span (Wechsler, 1981).

On the basis of the previous literature (Hampshire et al., 2012), the raw scores from each of the 12 subtests were $z$ score normalized using the mean and standard deviation of each subtest from a large $(N=44,600)$, young population. Each test item was then weighted according to the factor loadings from Hampshire et al. (2012), and then the respective subtests were averaged to create the Reasoning, STM, and Verbal subscores and transformed to a mean of 100 and an $S D$ of 15 so that test scores were readily comparable with results from similar studies by our group that employed test batteries tapping Reasoning and Verbal abilities such as the MAB-II (Fogel \& Smith, 2006; 2007). The descriptive statistics of each subscore are shown in Table 2.

\section{Sleep Quality Measures}

Participants' sleep quality was determined based on their sleep efficiency (SE) and the number of awakenings (NA) to assess sleep fragmentation, derived from the PSG re- cording night. SE was calculated as a percentage of the total time spent in bed between "lights off" and "lights on" divided by the time spent in bed actually sleeping. NA was defined as any 30-sec epoch, after sleep onset, that was scored as wake because of either (1) occipital EEG alpha activity occurring for more than $50 \%$ of the epoch or (2) a body movement as well as an alpha activity occurring for a part of the epoch (even $<50 \%$ of the epoch; Iber et al., 2007).

\section{Chronotype}

The Horne-Ostberg Morningness-Eveningness Questionnaire (MEQ; Horne \& Ostberg, 1976) was administered to all participants to assess their circadian rhythm type (e.g., morning, neutral, or evening chronotype). The MEQ is a paper-and-pencil test consisting of 19 questions that address items such as subjective assessment of intellectual and physical peak times, sleep/wake habits, and appetite and alertness over the course of the day. Total scores for the MEQ range from 16 (i.e., extreme-evening type) to 86 (i.e., extreme-morning type).

\section{Procedure}

All participants were initially screened to verify that they met the inclusion criteria (see Participants section for details). Each participant underwent two nights of PSG recordings including an initial acclimatization/screening night and, 2-7 days later, a PSG recording night (Figure 1). Immediately before the PSG recording night, all participants were asked to complete the Stanford Sleepiness Scale and Epworth Sleepiness Scale to determine their subjective sleepiness and the MEQ to determine the extent of their morningness-eveningness tendencies. After the PSG recording night, all participants completed the CBS Trials (see Intelligence testing section above for details) online in their own homes between the hours of 9:00 am and 9:00 pm.

\section{Data Analyses}

General Approach: Testing Mediation of the Relationship between Spindles and Cognitive Abilities by Sleep Quality and Morningness-Eveningness Tendency

Data from 24 of the 27 participants were included in the analyses (three participants have missing CBS data). All
Figure 1. Experimental protocol. Participants were screened for sleep quality and sleep disorders from an overnight $(\sim 11: 00 \mathrm{pm}$ to 7:00 am) PSG (see Slow spindles in NREM2 and SWS section). This also served as an opportunity to acclimatize participants to sleeping in the laboratory environment. Before the PSG recording night, participants completed the sleep questionnaires and the Horne-Ostberg MEQ. Two to seven days after the screening night, participants returned to the laboratory for a full-night PSG recording. After the PSG recording night, participants completed the CBS Trials online between the hours of 9:00 am and 9:00 pm.

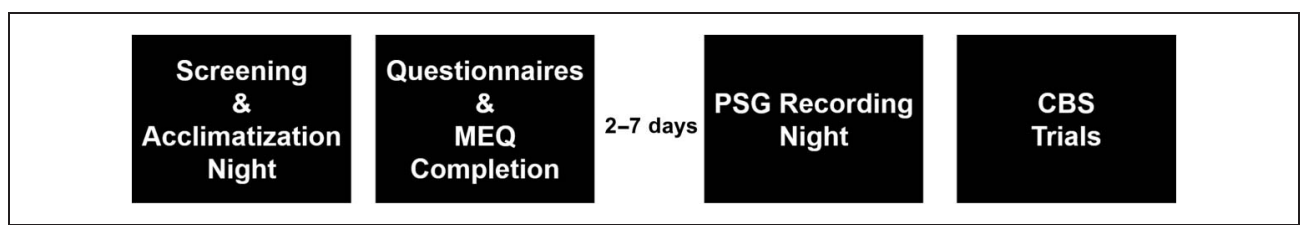




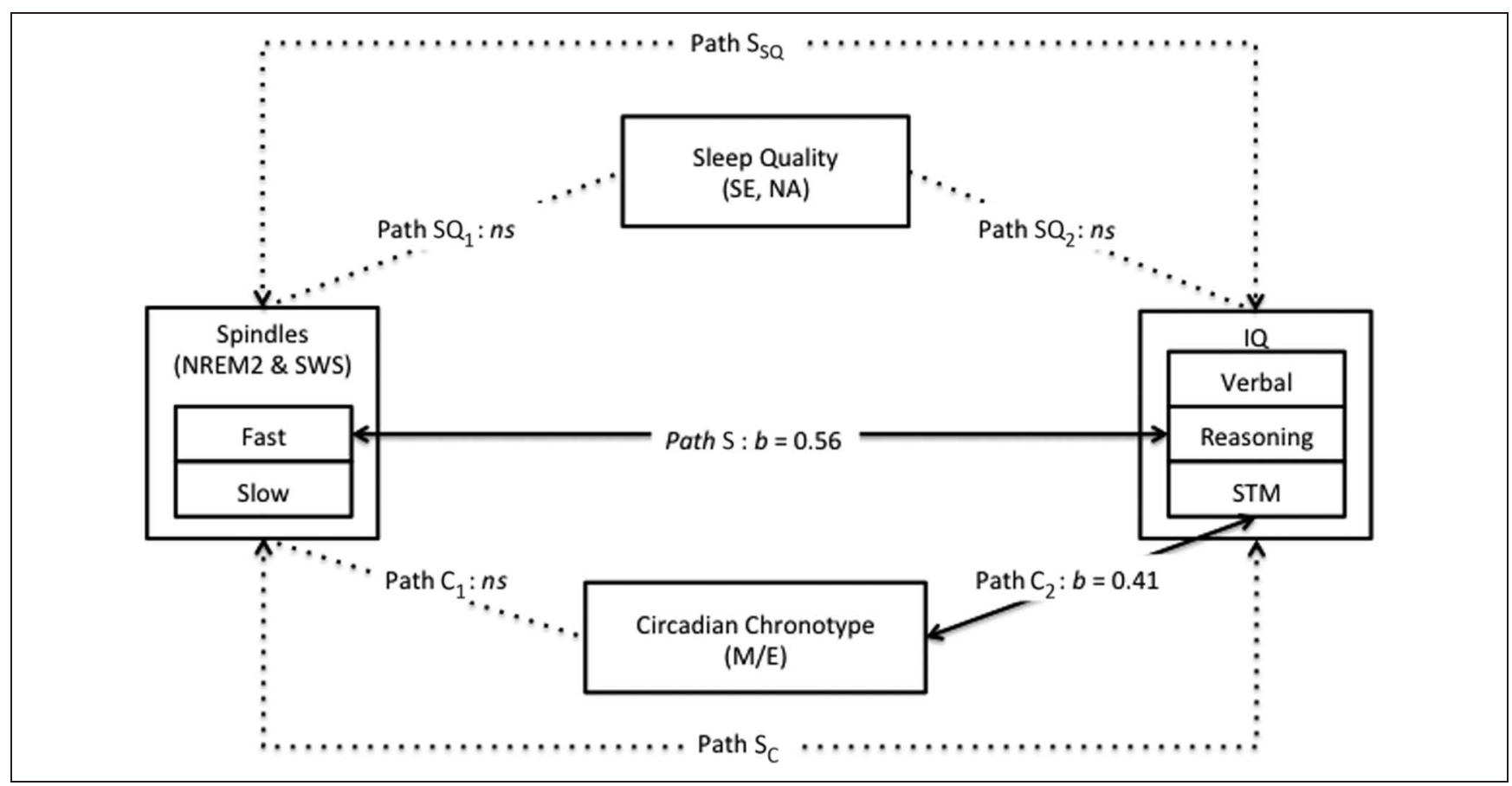

Figure 2. Statistical model. We tested the possibility that sleep quality or circadian chronotype (morningness-eveningness tendency) might partially or completely explain the relationship between spindles and cognitive abilities. There was no relationship between spindles and sleep quality (Path $\mathrm{SQ}_{1}$ ) or between sleep quality and cognitive abilities (Path $\mathrm{SQ}_{2}$ ), suggesting that the relationship between spindles and cognitive abilities was not mediated by sleep quality (Path $\mathrm{S}_{\mathrm{SQ}}$ ). In addition, there was no relationship between spindles and circadian chronotype (Path $\mathrm{C}_{1}$ ), and thus, circadian factors did not mediate the relationship between spindles and cognitive abilities (Path $\mathrm{S}_{\mathrm{C}}$ ). However, there was a significant relationship between circadian chronotype and STM but not reasoning or verbal abilities (Path $\mathrm{C}_{2}$ ), suggesting that circadian factors related to STM, but independently of spindles. Only fast, but not slow, sleep spindles during NREM2 and SWS were related to reasoning abilities, but not verbal or STM abilities, thus supporting the hypothesis that sleep spindles are directly related to reasoning abilities, independent of sleep quality or circadian factors. Note: Nonstatistically significant $(n s)$ results are indicated by dashed lines; significant results are indicated by solid arrows.

statistical analyses were carried out using SPSS Statistics version 22 (IBM, Armonk, NY). To test the hypothesis that sleep spindles were directly related to intellectual ability (Figure 2, Path S) and not indirectly related (i.e., mediated) by sleep quality (Figure 2, Path SQ) or morningnesseveningness tendency (Figure 2, Path C), a four-step process (Baron \& Kenny, 1986) is required. Step 1 tested whether a relationship existed between spindles and cognitive abilities (Figure 2, Path S). Step 2 tested for a relationship between spindles and either sleep quality (Figure 2, Path $\mathrm{SQ}_{1}$ ) or circadian (Figure 2, Path $\mathrm{C}_{1}$ ) factors (i.e., circadian chronotype). Step 3 tested for a relationship between cognitive abilities and sleep quality (Figure 2, Path $\mathrm{SQ}_{2}$ ) or circadian factors (Figure 2, Path $\mathrm{SQ}_{2}$ ). The purpose of Steps 1-3 is to establish that zero-order relationships exist among the various factors that may account for and partially explain the relationship between spindles and intellectual ability. If one or more of these relationships are not statistically significant, then mediation is not possible or statistically unlikely. However, if all three steps are significant, Step 4 tests for some form (either partial or full) of mediation by sleep quality (Figure 2, Path $\mathrm{S}_{\mathrm{SQ}}$ ) or circadian factors (Figure 2, Path $\mathrm{S}_{\mathrm{C}}$ ) on the relationship between spindles and cognitive abilities. All statistical results were considered significant at $p<.05$. For all mul- tiple regression analyses, gender was included (covaried out) as a variable of noninterest.

\section{Step 1: Relationship between Sleep Spindles and Intellectual Ability (Path S)}

Linear regression analysis was used to examine the effects of sleep spindles on intellectual abilities (Reasoning, STM, and Verbal abilities). Sleep spindle duration, amplitude, and density were entered into each model as the independent variables; Reasoning, STM, and Verbal subscale factors were entered into separate models as the dependent variables. In addition, inspection of the resulting semipartial correlation coefficients was conducted to identify which subscale (e.g., Reasoning, Verbal, or STM) accounted for the greatest proportion of unique interindividual variability for each spindle characteristic. Finally, if warranted, post hoc regression analyses were conducted to identify which of the subtests that compose each factor account for unique variability in spindle characteristics. The subtests were entered together in each model as the independent variables, and spindle characteristics were entered into separate models as the dependent variable to determine which subtest was driving any significant 
relationships between spindle characteristics and cognitive ability.

Step 2: Relationship between Sleep Spindles and Sleep Quality (Path $S Q_{1}$ ) or Morningness-Eveningness Tendency $\left(\right.$ Path $\left.C_{1}\right)$

A second set of regressions was conducted to explore the relationship between sleep spindles and sleep quality or morningness-eveningness tendency. Sleep spindle duration, amplitude, and density were entered into each model as the independent variables; sleep quality and morningness-eveningness (MEQ) tendency were entered into separate models as the dependent variables. If significant, these analyses would indicate that sleep spindles might relate to intellectual ability only partially, via sleep quality or morningness-eveningness tendency. Otherwise, sleep quality or circadian chronotype was unlikely to mediate or explain the relationship between spindles and cognitive abilities.

Step 3: Relationship between Sleep Quality (Path $S Q_{2}$ ) or Morningness-Eveningness Tendency (Path $C_{2}$ ) on Intellectual Ability

Linear regression analysis was used to test for a mediation effect of sleep quality or morningness-eveningness tendency on the relationship between spindles and cognitive abilities. For sleep quality, we entered sleep quality measures (SE and NA) into each model as the independent variables and intellectual ability factor subscales (reasoning, STM, and verbal) into separate models as the dependent variables. The same approach was used to test the relationship between each ability factor and morningness-eveningness (MEQ) tendency.

Step 4: Mediation Effects of Sleep Quality and Circadian Morningness-Eveningness Tendency on the Relationship between Sleep Spindles and Intellectual Ability (Paths $S_{S Q}$ and $S_{C}$ )

Finally, if significant relationships were identified for Steps 1-3, we planned to examine the mediation effects of sleep quality and morningness-eveningness tendency on the relationship between sleep spindles and intellectual ability (e.g., full mediation, partial mediation, or no mediation). Separate linear regression analyses were planned with sleep spindle characteristics (duration, amplitude, and density), sleep quality measures (SE and NA) entered as the independent variables in one set of models, and morningness-eveningness (MEQ) tendency entered as an independent variable in another. Intellectual ability factor subscales (reasoning, STM, and verbal) were entered as the dependent variables into separate models. If the effect of spindles on intellectual ability was reduced, but still significant, this would indicate a partial mediation effect of sleep quality or cir- cadian chronotype. If the effect of spindles on intellectual ability was no longer significant, it would indicate a full mediation effect of sleep quality or circadian chronotype and that sleep spindles were, in fact, not directly related to cognitive abilities. If, however, the effect of spindles on intellectual ability did not change, it would indicate that spindles were directly related to cognitive abilities, independent of sleep quality or circadian factors.

\section{RESULTS}

\section{Fast Spindles}

\section{Fast Spindles during NREM2}

Step 1: Relationship between fast spindles during NREM2 and intellectual ability (Path S). Standard multiple linear regression analysis revealed that, taken together, the duration, amplitude, and density of fast spindles during NREM2 significantly accounted for variability in reasoning abilities $(F(3,20)=3.391, p=.030)$. Inspection of the semipartial coefficients revealed that the amplitude of fast spindles uniquely accounted for variability in reasoning abilities over and above duration and density $(t(20)=$ $2.340, b=0.501, p=.030$; Figure $3 \mathrm{~A})$. Fast spindle density $(t(20)=1.383, b=0.260, p=.183)$ and duration $(t(20)=$ $-1.922, b=-0.393, p=.070$ ) did not significantly account for variability in reasoning abilities, although it is worth noting that these effects did approach statistical significance. There were no statistically significant results for fast spindles for either STM or verbal abilities (Table 3), therefore suggesting that fast spindles during NREM2 sleep were related to reasoning abilities but not to STM or verbal abilities.

Post hoc analyses were conducted to determine which reasoning ability subtests were related to fast spindles during NREM2 sleep. Fast spindle characteristics together accounted for variability in deductive reasoning and the interlocking polygons subtest (Table 3). More specifically, fast spindle amplitude $(t(20)=3.510, p=.003)$ and duration $(t 20)=-3.806, p=.001)$ uniquely accounted for variability in deductive reasoning over and above spindle density (Figure 3B and C). Spindle density, amplitude, and duration together also accounted for variability on the interlocking polygons subtest only at a statistical trend level $(F(3,20)=2.482, p=.079)$. Taken together, these results suggest that interindividual differences in the size of sleep spindles (i.e., larger amplitude but shorter spindles) most closely relate to deductive reasoning abilities.

Step 2: Relationship between fast spindles during NREM2 with sleep quality (Path $S Q_{1}$ ) and morningness-eveningness tendency $\left(\right.$ Path $\left.C_{1}\right)$

Path $S_{1}$ : Effects of sleep spindles on sleep quality. Measures of sleep quality (SE and NA) were included in 


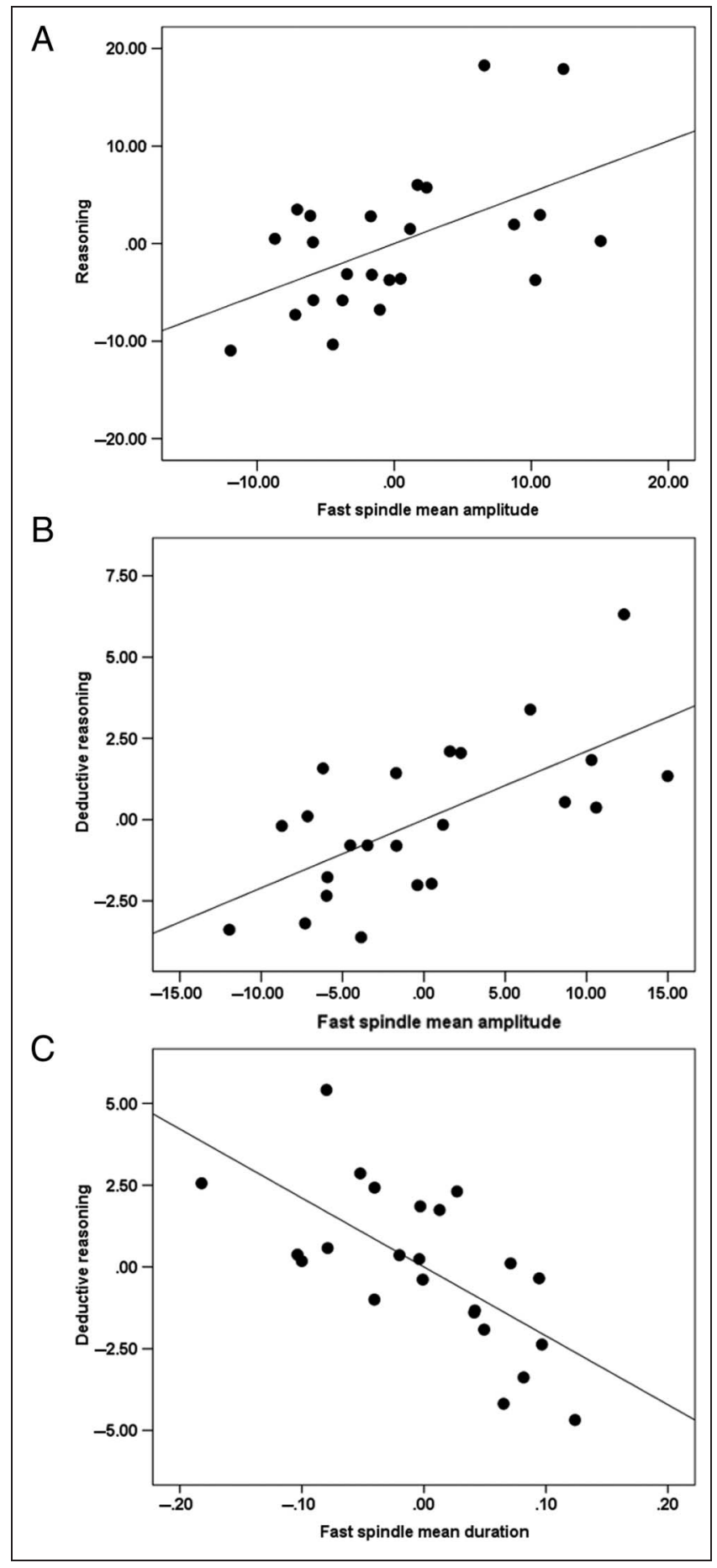

Figure 3. Fast spindles during NREM2 and reasoning. The unique relationship (i.e., semipartial correlations) between (A) fast spindle amplitude, over and above spindle duration and density, during NREM2 and reasoning abilities (standardized coefficient $b=0.501, p=.03$ ). (B) Fast spindle amplitude (standardized coefficient $b=0.683, p=.003$ ) and (C) duration (standardized coefficient $b=-0.704, p=.001$ ) uniquely accounted for variability in deductive reasoning over and above spindle density. the models as the dependent variables, and spindle characteristics (duration, amplitude, and density) were included together in each model as the independent variables. As shown in Table 4, no relationship was observed between fast spindles during NREM2 and any measure of sleep quality.

Path $C_{1}$ : Effects of sleep spindles on circadian morningnesseveningness. Circadian chronotype (i.e., MEQ scores) was included in the models as the dependent variable, and spindle characteristics (duration, amplitude, and density) were included together in each model as the independent variables. As shown in Table 4, no relationship was observed between fast spindles and MEQ scores.

Taken together, the results of Step 2 revealed that fast spindles during NREM2 had no direct relationship with sleep quality or circadian chronotype, indicating that the relationship between fast spindles and reasoning abilities was independent of sleep quality and circadian chronotype. Therefore, it was not warranted to conduct Step 4 (Paths $\mathrm{S}_{\mathrm{SQ}}$ and $\mathrm{S}_{\mathrm{C}}$ ) to test for a mediation effect of sleep quality and morningness-eveningness tendency on the relationship between spindles and cognitive abilities. A comparison of the resulting coefficient values ( $b$ value) between Steps 1 and 4 can be found in the supplement (Table 5). We did conduct Step 3 of the analysis to examine the effects of sleep quality (Path $\mathrm{SQ}_{2}$ ) and morningnesseveningness tendency (Path $\mathrm{C}_{1}$ ) on intellectual ability in the Sleep quality and morningness-eveningness tendency section.

\section{Fast Spindles during SWS}

Step 1: Relationship between fast spindles during SWS and intellectual ability (Path S). The same regression approach employed in the fast spindles during NREM2 section was used to investigate the relationship between fast spindles during SWS and intellectual ability. Similar to fast spindles during NREM2, the standard multiple linear regression analysis revealed that, taken together, the duration, amplitude, and density of fast spindles during SWS significantly accounted for variability in reasoning abilities $(F(3,20)=3.022, p=.040 ;$ Table 6$)$. Inspection of the semipartial coefficients revealed that both amplitude $(t(20)=$ $2.386, b=0.572, p=.028$; Figure $4 \mathrm{~A})$ and density $(t(20)=$ $1.996, b=0.452, p=.040$; Figure $4 \mathrm{~B}$ ) of fast spindles during SWS accounted for variability in reasoning abilities over and above duration. Fast spindle duration did not significantly account for variability in reasoning abilities $(t(20)=-1.126, b=-0.256, p=.274)$. There were no statistically significant results for fast spindles for either STM or verbal abilities (see Table 6). Therefore, similar to NREM2 spindles, the amplitude of fast spindles during SWS sleep was related to reasoning abilities but not to STM or verbal abilities.

Post hoc analyses were conducted to determine which reasoning ability subtests were related to fast spindles 
Table 3. Multiple Regression Analyses of the Relationship between CBS Trials and Fast Spindles during NREM2

\begin{tabular}{|c|c|c|c|c|c|c|c|c|c|c|c|c|}
\hline \multirow[b]{2}{*}{ IQ Measures } & \multicolumn{3}{|c|}{ Overall Effect } & \multirow[b]{2}{*}{$t$} & \multicolumn{3}{|c|}{ Amplitude } & \multicolumn{3}{|c|}{ Density } & \multicolumn{2}{|c|}{ Duration } \\
\hline & $F(3,20)$ & $s r^{2}$ & $p$ & & $b$ & $p$ & $t$ & $b$ & $p$ & $t$ & $b$ & $p$ \\
\hline Reasoning & 3.391 & 0.417 & $.030 *$ & 2.340 & 0.501 & $.030 *$ & 1.383 & 0.260 & .183 & -1.922 & -0.393 & .070 \\
\hline Deductive reasoning & 5.398 & 0.545 & $.005 *$ & 3.510 & 0.683 & $.003 *$ & 1.060 & 0.180 & .303 & -3.806 & -0.704 & $.001 *$ \\
\hline Spatial rotation & 1.265 & 0.210 & .318 & & & & & & & & & \\
\hline Feature match & 0.800 & 0.151 & .541 & & & & & & & & & \\
\hline Spatial planning & 0.958 & 0.131 & .433 & & & & & & & & & \\
\hline Interlocking polygons & 2.482 & 0.343 & .079 & & & & & & & & & \\
\hline STM & 0.180 & 0.037 & .946 & & & & & & & & & \\
\hline Verbal & 1.500 & 0.240 & .242 & & & & & & & & & \\
\hline
\end{tabular}

Statistically significant results indicated by an asterisk $(*)$ at $p<.05$.

during SWS (Table 6). Similar to fast spindles in NREM2, the deductive reasoning subtest with spindle amplitude uniquely correlates with deductive reasoning (Figure $4 \mathrm{C}$ ) over and above spindle density and duration.

Step 2: Relationship between fast spindles during SWS with sleep quality (Path $S Q_{1}$ ) and morningnesseveningness tendency (Path $C_{1}$ )

Path $S Q_{1}$ : Effects of sleep spindles on sleep quality. The same regression approach as employed in Step 2 under the fast spindles during NREM2 section for fast spindles during NREM2 was used to examine the relationship between fast spindles during SWS and sleep quality. As shown in Table 4, no significant relationship was observed between fast spindles during SWS and any measure of sleep quality.

Path $C_{1}$ : Effects of sleep spindles on morningnesseveningness tendency. Similarly, the same regression approach as employed in Step 2 under the fast spindles during NREM2 section for fast spindles during NREM2 was used to examine the relationship between fast spindles during SWS and morningness-eveningness tendency. As shown in Table 4, no significant relationship was observed between fast spindles during SWS and morningnesseveningness tendency.

Similar to fast spindles during NREM2, taken together, the results of Step 2 revealed that fast spindles during SWS had no direct effect on sleep quality or morningnesseveningness tendency, indicating that the effects of fast spindles on reasoning abilities were independent of sleep quality or circadian chronotype. Therefore, it was not warranted to conduct Step 4 (Paths $\mathrm{S}_{\mathrm{SQ}}$ and $\mathrm{S}_{\mathrm{C}}$ ) to test for a mediation effect of sleep quality and morningnesseveningness tendency on the relationship between spindles and cognitive abilities. As noted above, we did conduct Step 3 of the analysis to examine the effects of sleep quality (Path $\mathrm{SQ}_{2}$ ) and morningness-eveningness tendency (Path $\mathrm{C}_{1}$ ) on intellectual ability in the Sleep quality and morningness-eveningness tendency section.

Sleep Quality and Morningness-Eveningness Tendency Step 3: Relationship between sleep quality (Path $S_{2}$ ) or morningness-eveningness tendency (Path $C_{2}$ ) and intellectual ability

Path $\mathrm{SQ}_{2}$ : Relationship between sleep quality and intellectual ability. A linear multiple regression analysis was

Table 4. Multiple Regression Analyses of the Relationship between Sleep Quality and Chronotype with Fast and Slow Spindles during NREM2 and SWS

\begin{tabular}{|c|c|c|c|c|c|c|c|c|c|c|c|c|}
\hline & \multicolumn{6}{|c|}{ Fast Spindles } & \multicolumn{6}{|c|}{ Slow Spindles } \\
\hline & \multicolumn{3}{|c|}{ NREM2 } & \multicolumn{3}{|c|}{ SWS } & \multicolumn{3}{|c|}{ NREM2 } & \multicolumn{3}{|c|}{ SWS } \\
\hline & $F(3,20)$ & $s r^{2}$ & $p$ & $F(3,20)$ & $s r^{2}$ & $p$ & $F(3,20)$ & $s r^{2}$ & $p$ & $F(3,20)$ & $s r^{2}$ & $p$ \\
\hline SE & 1.117 & 0.169 & .374 & 1.112 & 0.168 & .376 & 1.705 & 0.237 & .185 & 1.738 & 0.240 & .178 \\
\hline NA & 2.121 & 0.278 & .112 & 2.375 & 0.302 & .083 & 2.170 & 0.283 & .106 & 2.170 & 0.283 & .106 \\
\hline MEQ & 0.868 & 0.136 & .499 & 0.361 & 0.062 & .834 & 0.801 & 0.127 & .538 & 0.768 & 0.123 & .557 \\
\hline
\end{tabular}

NREM2 = NREM sleep stage 2; SWS = slow wave sleep; SE = sleep efficiency; NA = number of awakenings; MEQ = Horne-Ostberg MorningnessEveningness Questionnaire. 


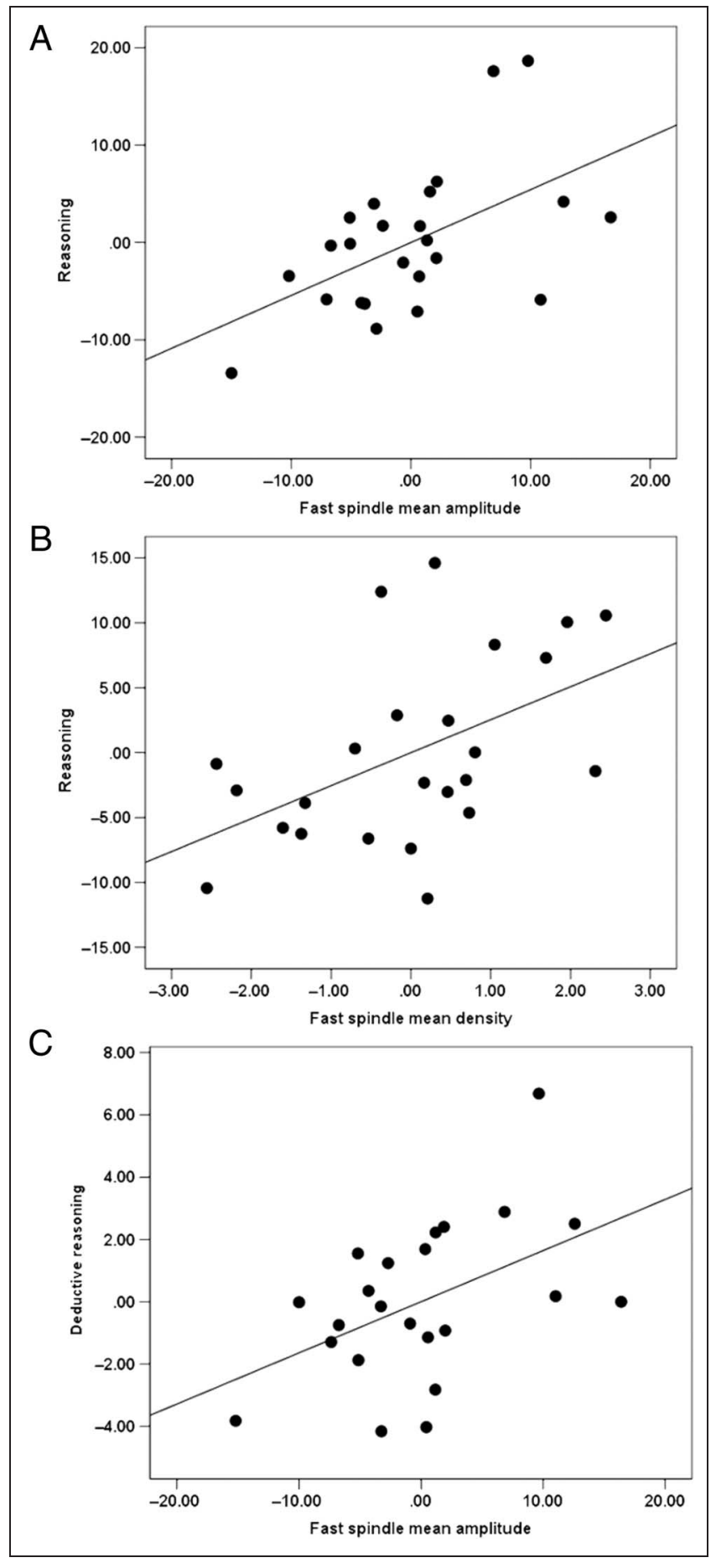

Figure 4. Fast spindles during SWS and reasoning. The unique relationship (i.e., semipartial correlation) between (A) fast spindle amplitude (over and above duration and density) during SWS and reasoning abilities (standardized coefficient $b=0.572, p=.028$ ) and (B) the unique relationship between fast spindle density (over and above duration and amplitude) during SWS and reasoning abilities (standardized coefficient $b=0.452, p=.04$ ) and (C) amplitude (standardized coefficient $b=0.609, p=.031$ ) uniquely accounted for variability in deductive reasoning over and above spindle density.
Table 5. Coefficient Value Comparison between Step 1 and Step 4

\begin{tabular}{|c|c|c|c|c|c|c|}
\hline \multirow[b]{3}{*}{ Reasoning } & \multicolumn{6}{|c|}{ NREM2 } \\
\hline & \multicolumn{2}{|c|}{ Amplitude } & \multicolumn{2}{|c|}{ Density } & \multicolumn{2}{|c|}{ Duration } \\
\hline & b1 & $p 1$ & b1 & p1 & b1 & $p 1$ \\
\hline & 0.501 & .030 & 0.260 & .183 & -0.393 & .070 \\
\hline & $b 2$ & $p 2$ & $b 2$ & $p^{2}$ & $b 2$ & $p 2$ \\
\hline & 0.487 & .040 & 0.348 & .101 & -0.434 & .055 \\
\hline \multirow[t]{3}{*}{$\begin{array}{l}b \text {-Value } \\
\text { comparison }\end{array}$} & \multicolumn{2}{|c|}{0.95} & \multicolumn{2}{|c|}{0.76} & \multicolumn{2}{|c|}{0.87} \\
\hline & \multicolumn{6}{|c|}{ SWS } \\
\hline & \multicolumn{2}{|c|}{ Amplitude } & \multicolumn{2}{|c|}{ Density } & \multicolumn{2}{|c|}{ Duration } \\
\hline \multirow[t]{4}{*}{ Reasoning } & b1 & $p 1$ & b1 & $p 1$ & b1 & $p 1$ \\
\hline & 0.572 & .028 & 0.452 & .040 & -0.256 & .274 \\
\hline & b2 & $p^{2}$ & b2 & $p^{2}$ & b2 & $p 2$ \\
\hline & 0.569 & .044 & 0.490 & .050 & -0.257 & .324 \\
\hline $\begin{array}{l}b \text {-Value } \\
\quad \text { comparison }\end{array}$ & \multicolumn{2}{|c|}{0.98} & \multicolumn{2}{|c|}{0.87} & \multicolumn{2}{|c|}{0.99} \\
\hline
\end{tabular}

(1) b1 is the standardized regression coefficient, and p1 is the respective statistical significance of each spindle feature in the regression model of Step 1 (including spindle amplitude, density, and duration as independent variables). (2) b2 indicates the standardized regression coefficient, and $\mathrm{p} 2$ indicates the respective statistical significance of each spindle feature in the regression model of Step 4 (including each spindle feature, sleep quality, and circadian chorotype [MEQ] as independent variables). (3) We then calculated the statistical significance of the difference (" $b$-value comparison") between the two coefficients (b1 and b2) to examine whether inclusion of sleep quality and circadian chorotype in the models would influence the correlation between spindles and reasoning ability. Results show that when sleep quality and circadian chorotype are included in the model, the standard coefficient values from Step 1 did not change significantly, which provides further support that sleep spindles are directly related to reasoning ability.

conducted to examine the relationship between sleep quality and intellectual ability. Intellectual ability scores (reasoning, STM, and verbal) were included in each model as the dependent variables, and measures of sleep quality (SE and NA) were included in the separate models as the independent variables. As shown in Table 7 , no significant relationship was found between any measure of sleep quality and any intellectual ability.

Path $C_{2}$ : Relationship between morningness-eveningness tendency and intellectual ability. A similar approach was used to investigate the relationship between circadian chronotype (i.e., MEQ scores) and intellectual ability scores (reasoning, STM, and verbal). STM was the only intellectual ability correlated with MEQ scores $(t(22)=$ $-2.131, b=-0.414, p=.044$; Table 8 and Figure 5), indicating that evening types had higher STM scores than morning types. No relationship was observed between 
Table 6. Multiple Regression Analyses of the Relationship between CBS Trials and Fast Spindles during SWS

\begin{tabular}{|c|c|c|c|c|c|c|c|c|c|c|c|c|}
\hline \multirow[b]{2}{*}{ IQ Measures } & \multicolumn{3}{|c|}{ Overall Effect } & \multirow[b]{2}{*}{$t$} & \multicolumn{3}{|c|}{ Amplitude } & \multicolumn{3}{|c|}{ Density } & \multicolumn{2}{|c|}{ Duration } \\
\hline & $F(3,20)$ & $s r^{2}$ & $p$ & & $b$ & $p$ & $t$ & $b$ & $p$ & $t$ & $b$ & $p$ \\
\hline Reasoning & 3.022 & 0.389 & $.040^{*}$ & 2.386 & 0.572 & $.028^{*}$ & 1.996 & 0.452 & $.040 *$ & -1.126 & -0.256 & .274 \\
\hline Deductive reasoning & 2.008 & 0.308 & .137 & 2.335 & 0.609 & $.031 *$ & 1.761 & 0.432 & .095 & -1.436 & -0.352 & .168 \\
\hline Spatial rotation & 1.934 & 0.289 & .146 & & & & & & & & & \\
\hline Feature match & 1.293 & 0.223 & .310 & & & & & & & & & \\
\hline Spatial planning & 1.833 & 0.289 & .167 & & & & & & & & & \\
\hline Interlocking polygons & 1.501 & 0.240 & .241 & & & & & & & & & \\
\hline STM & 0.138 & 0.028 & .966 & & & & & & & & & \\
\hline Verbal & 0.740 & 0.135 & .577 & & & & & & & & & \\
\hline
\end{tabular}

Statistically significant results indicated by an asterisk (*) at $p<.05$.

MEQ scores and reasoning abilities $(t(22)=-0.393, b=$ $-0.084, p=.697)$ or verbal abilities $(t(22)=-0.219, b=$ $-0.047, p=.828)$.

\section{Slow Spindles}

\section{Slow Spindles in NREM2 and SWS}

We conducted the same multiple regression analyses as for fast spindles as reported in the Fast spindles section to investigate whether sleep quality or circadian chronotype mediated the relationship between slow spindles and intellectual abilities. However, there were no statistically significant results for any analyses (Tables 4 and 8).

Taken together, these results suggest that fast, but not slow, spindles in both NREM2 and SWS are directly related to reasoning abilities, but not to verbal abilities or STM, and are not mediated by sleep quality or circadian factors. Deductive reasoning, reflecting fluid intelligence, was most consistently and uniquely related to spindle amplitude.

\section{Fast Versus Slow Spindles in NREM2 and SWS}

To follow up if significant correlations between spindles and cognitive abilities (Table 9) differed for fast and slow

Table 7. Multiple Regression Analyses of the Relationship between CBS Trials and Measures of Sleep Quality and Circadian Chronotype (Morningness-Eveningness)

\begin{tabular}{lcccccccc}
\hline & \multicolumn{3}{c}{ Sleep Quality } & & \multicolumn{3}{c}{ Circadian Chronotype } \\
\cline { 2 - 3 } IQ Measures & $F(2,21)$ & $s r^{2}$ & $p$ & & $F(1,22)$ & $s r^{2}$ & $p$ \\
\hline Reasoning & 1.529 & 0.187 & .238 & 2.608 & 0.199 & .097 \\
STM & 0.084 & 0.012 & .968 & 4.543 & 0.171 & $.034^{*}$ \\
Verbal & 0.823 & 0.110 & .497 & & 0.562 & 0.051 & .578 \\
\hline
\end{tabular}

Statistically significant results indicated by an asterisk (*) at $p<.05$. spindles within a particular stage of sleep and to compare correlations between NREM2 and SWS, we computed the 95\% confidence intervals about each regression coefficient for fast and slow spindles in both NREM2 and SWS (Table 9). This follow-up procedure revealed that the relationship between fast spindle amplitude and reasoning ability was significantly stronger than that for slow spindles in NREM2. The same pattern of results was observed for fast spindle amplitude, density, and duration in SWS. In addition, there were no differences for each spindle type (slow or fast) when directly comparing across NREM sleep stages (NREM2 vs. SWS).

\section{DISCUSSION}

Sleep spindles are phasic EEG hallmark features of NREM sleep (both NREM2 and SWS) whose characteristics are very trait like and stable from night to night within an individual but vary considerably between individuals. The functional significance and biological basis of these interindividual differences remain to be fully elucidated; however, the relationship between spindles and intellectual ability is well established across numerous studies employing various but related tests including the MAB-II (Fogel et al., 2007; Fogel \& Smith, 2006; Nader \& Smith, 2001, 2003), the Raven's Standard and Advanced Progressive Matrices Tests (Ujma et al., 2014; Schabus et al., 2006; Bódizs et al., 2005), the Wechsler Memory Scale-Revised (Schabus et al., 2006), and the Cattell's Culture Fair Intelligence Test (Ujma et al., 2014). Importantly, sleep spindles are also associated with sleep maintenance (Schabus et al., 2012; Dang-Vu et al., 2010; Vyazovskiy et al., 2004; Cote et al., 2000; Elton et al., 1997; Steriade, 1991) and circadian rhythmicity (Dijk \& Cajochen, 1997; Dijk \& Czeisler, 1995; Dijk et al., 1993, 1995). In addition, sleep quality (Nebes et al., 2009; Blackwell et al., 2006; Achilles, 2003; Bastien et al., 2003; Hauri, 1997; Bonnet \& Arand, 1995) and circadian rhythmicity (Achilles, 2003; Taillard, Philip, Coste, Sagaspe, \& Bioulac, 2003; Song \& Stough, 2000; 
Table 8. Multiple Regression Analyses of the Relationship between CBS Tests and Slow Spindles during NREM2 and SWS

\begin{tabular}{|c|c|c|c|c|c|c|}
\hline & \multicolumn{3}{|c|}{ Slow Spindles during NREM2 } & \multicolumn{3}{|c|}{ Slow Spindles during SWS } \\
\hline & $F(3,20)$ & $s r^{2}$ & $p$ & $F(3,20)$ & $s r^{2}$ & $p$ \\
\hline Reasoning & 1.018 & 0.176 & .423 & 1.332 & 0.219 & .294 \\
\hline STM & 0.275 & 0.055 & .890 & 0.264 & 0.053 & .898 \\
\hline Verbal & 0.254 & 0.051 & .903 & 0.617 & 0.115 & .656 \\
\hline
\end{tabular}

Roberts \& Kyllonen, 1999; Dijk \& Cajochen, 1997; Dijk \& Czeisler, 1995; Dijk et al., 1993, 1995) are also associated with cognitive abilities. Considering the interrelationship between spindles, intellectual abilities, sleep maintenance, sleep quality, and circadian-related factors, it is possible that one or more of these related aspects may either partially or entirely explain the correlation between spindles and cognitive abilities. To our knowledge, no study to date has specifically tested the contribution of these other factors to the relationship between spindles and cognitive abilities in the same set of individuals. Here, using a combination of cognitive testing, objective and subjective measures of sleep quality, and circadian chronotype (i.e., morningness-eveningness tendencies), we aimed to explore if the relationship between sleep spindles and intellectual ability is a direct effect or whether it is mediated by sleep quality or other trait-like factors such as circadian chronotype.

In general, our results support previous studies demonstrating that sleep spindles are associated with reasoning abilities but not verbal or STM abilities (Fogel et al., 2007; Schabus et al., 2006; Bódizs et al., 2005; Nader \& Smith, 2001, 2003). Similar to previous studies (Ray et al., 2015; Zeitlhofer et al., 1997; Dijk et al., 1993), we found that the characteristics of sleep spindles differed between NREM2 and SWS. However, the functional sig-

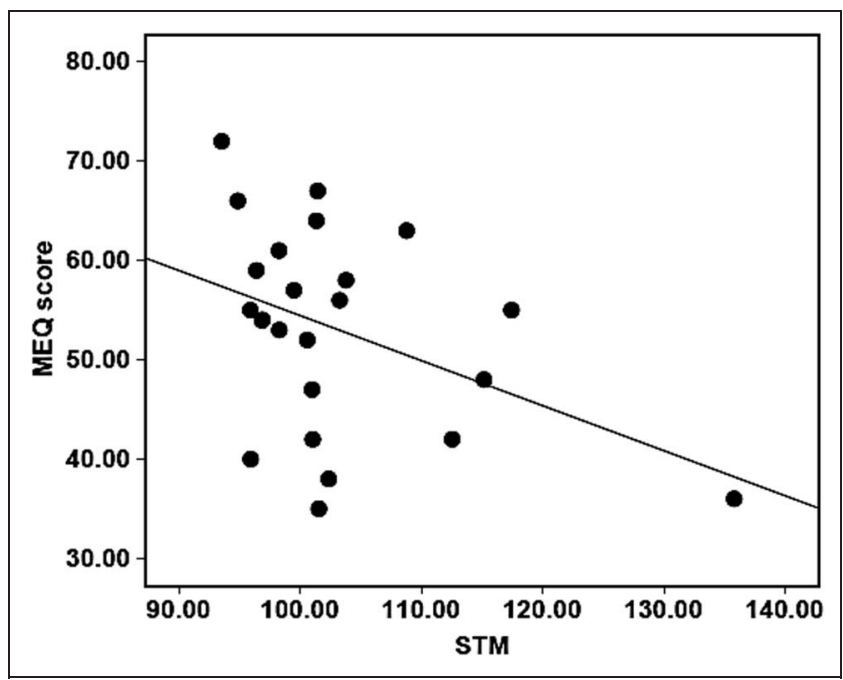

Figure 5. The relationship between scores on the MEQ and STM abilities $(r=-.414, p<.05)$. nificance of these differences is not yet well understood. Here, we found that the amplitude of fast spindles during both NREM2 and SWS sleep was related to reasoning abilities, but not to STM or verbal abilities. In addition, the density of fast spindles during SWS sleep was related to reasoning abilities. No relationship was found between fast spindles and verbal or STM abilities in either NREM2 or SWS. By contrast, slow spindles were not related to any cognitive ability. Although the characteristics of sleep spindles differed between NREM2 and SWS, follow-up tests revealed that the relationship between spindles and reasoning abilities was similar in NREM2 and SWS. This suggests that, although spindles differ in terms of their form in NREM2 versus SWS, they may support intellectual functioning in a similar manner irrespectively if they occur in NREM2 or SWS. In addition, the relationship between fast spindle characteristics and reasoning abilities was stronger than slow spindles in both NREM2 and SWS. Thus, taken together, these results suggest that fast spindles were more strongly related to reasoning abilities than slow spindles, regardless of whether they Occur in NREM2 or SWS, and that slow spindles may serve a dissociable, and still yet to be determined, function from fast spindles. Further analyses revealed that, among the subtests that comprise the Reasoning factor, the amplitude of fast spindles during both NREM2 sleep and SWS was positively related to fluid intelligence as reflected by the correlation with performance on the deductive reasoning subtest.

Importantly, neither sleep quality nor morningnesseveningness tendency was significantly correlated with spindles and therefore did not mediate the relationship between reasoning abilities and fast sleep spindles in NREM2 and SWS. Thus, this study demonstrates, for the first time, that the relationship between spindles and cognitive abilities is a direct one, which is not confounded or mediated by extraneous factors that modulate spindles such as sleep quality or circadian chronotype. Taken together, this suggests that, regardless of the NREM sleep stage for which the spindles occur, fast sleep spindles, but not slow spindles, are uniquely related to reasoning abilities, over and above sleep quality and circadian chronotype, and may serve as a biological marker for intellectual abilities assessed by tests that tap into the capacity for reasoning (i.e., to identify complex patterns and relationships and the use of logic; Fogel et al., 2007). 
Table 9. Partial Correlation ( $\pm 95 \%$ Confidence Interval) between Spindles and Reasoning Ability

\begin{tabular}{|c|c|c|c|c|}
\hline & \multicolumn{2}{|c|}{ NREM2 } & \multicolumn{2}{|c|}{ SWS } \\
\hline & Fast Spindles & Slow Spindles & Fast Spindles & Slow Spindles \\
\hline & \multicolumn{2}{|c|}{ Partial Correlation (r) } & \multicolumn{2}{|c|}{ Partial Correlation (r) } \\
\hline Amplitude & $.52[.15, .77]^{*}$ & $.09[-.33, .48]$ & $.48[.09, .74]^{*}$ & $-.13[-.51, .29]$ \\
\hline Density & $.37[-.04, .68]$ & $.02[-.39, .43]$ & $.42[.01, .70]^{*}$ & $-.09[-.48, .33]$ \\
\hline Duration & $-.42[-.71,-.02]$ & $-.10[-.49, .32]$ & $-.25[-.60, .17]^{*}$ & $.22[-.21, .57]$ \\
\hline
\end{tabular}

An asterisk (*) indicates significantly different from slow spindles, at $p<.05$. There were no differences between spindles in NREM2 and SWS

The CBS Trials test battery is advantageous as it is one of the only widely used cognitive test batteries for which the neural substrates supporting these distinct abilities are known. Hampshire et al. (2012) identified a distinct network of "multiple-demand" brain regions including the inferior frontal sulcus, inferior parietal cortex, and dorsal portion of ACC and pre-SMA whose activation explains interindividual differences in reasoning abilities. This was dissociable from a more frontally focused network of brain regions that are related to STM, including the insula, superior frontal sulcus, and ventral ACC/ pre-SMA. Verbal abilities, on the other hand, related most closely to a left-lateralized network including the left inferior frontal gyrus and bilateral temporal lobes. Interestingly, Schabus et al. (2007) identified an overlapping set of brain regions spontaneously activated by fast sleep spindles including ACC and SMA (Schabus et al., 2007). Although speculative, taken together, this suggests that reasoning abilities might be emergent properties of distinct networks of brain regions that are implicated in cognitive abilities during wakefulness but are also detectable during sleep in relation to the action of sleep spindles. At the cellular network level, a large body of studies employing multiunit recordings in animals and computational models has shown that spindles reflect oscillatory activity in widespread thalamocortical (TC) circuits. Although the neural circuitry that supports spindle generation is well known (Steriade, 2005) and involves a complex interaction between reticular (RE), TC, and pyramidal cells, more recent experimental evidence (Bonjean et al., 2011) suggests that corticothalamic input initiates spindles by triggering spike bursts in RE thalamic neurons and is maintained by synchronized firing in the RE-TC-RE circuit (Steriade, Nunez, \& Amzica, 1993; von Krosigk, Bal, \& McCormick, 1993). Spindles are terminated by desynchronization of the interaction between thalamic and cortical neuronal firing. Thus, the cortex plays a central role in both the initiation and termination of spindle events, influencing the foci and characteristics of spindles. This cortical involvement could have widespread implications for cognitive processes such as cognitive abilities and the maintenance of knowledge, and thus, normal and efficient generation of spindles may support normal intellectual abilities. Optimal or highly efficient generation of spindles may support highly developed cognitive aptitudes, as suggested by a very high and positive correlation between spindles and IQ in individuals in the gifted range of IQ scores (Fogel et al., 2007). On the other hand, deficient or dysfunctional spindle generation may be associated with compromised intellectual functioning. For example, Gibbs and Gibbs (1962) suggested that children with mental disability had abnormally large spindles, which they termed "extreme spindles." These abnormally high-amplitude and long-duration spindles were suggested to be the result of deficient gating mechanisms of TC circuitry (Bixler \& Rhodes, 1968). In addition, reduced spindle activity in those with schizophrenia was associated with cognitive deficits (Wamsley et al., 2012). Interestingly, the administration of eszopiclone during sleep significantly increased spindle generation in individuals with schizophrenia and the increases correlated with overnight motor sequence task improvement (Wamsley et al., 2013). Taken together, these results suggest that individual differences in the degree to which there is optimal functioning of the TC system may dictate and support normal intellectual abilities (for a review, see Fogel \& Smith, 2011). In the current study, our findings further demonstrate that spindle activity is directly linked with reasoning abilities independent of sleep quality and circadian influences, thus suggesting that the oscillatory action of spontaneous spindles per se, particularly in brain regions that support reasoning abilities, for example, in multiple-demand associative areas of the cortex (Hampshire et al., 2012), may support reasoning abilities. However, this possibility remains to be directly investigated.

Although no significant relationship was identified between measures of sleep quality and cognitive abilities, it was observed that circadian chronotype was associated with STM, whereby evening types had higher STM scores. These results are consistent with studies indicating that evening types are more likely to perform well on memory tasks (Roberts \& Kyllonen, 1999); however, this finding has not yet been reliably demonstrated (Schmidt, Peigneux, \& Cajochen, 2012; Hidalgo et al., 2004). Song and Stough (2000) found that morning types performed significantly worse in the morning in a spatial subtest of the MAB-II and performed better in the late afternoon. Conversely, evening types performed better in the morning than in 
the late afternoon. Roberts and Kyllonen (1999) also found that evening types were more likely to have higher intelligence scores, especially in working memory performance. Our results suggest that circadian chronotype relates to cognitive abilities that rely on abilities involving STM. It is also possible that circadian influences impact brain regions that support STM including the insula, superior frontal sulcus, and ventral ACC/pre-SMA (Hampshire et al., 2012). However, given the inconsistencies in the literature, further research is required to more clearly establish a link between circadian factors and cognitive abilities.

Surprisingly, we did not find any relationship between sleep spindles and sleep quality or morningness-eveningness tendency. On the basis of the protective function of spindles for sleep maintenance, by modulating external information processing during sleep (Schabus et al., 2012; Dang-Vu et al., 2010; Vyazovskiy et al., 2004; Cote et al., 2000; Elton et al., 1997; Steriade, 1991), we would have expected to observe a correlation between sleep spindles and metrics of sleep quality. In addition, given that morning types but not evening types have been found to have higher sigma power $(12-14 \mathrm{~Hz})$ during sleep (Mongrain et al., 2005), we would have expected there to be some correlation between spindles and morningnesseveningness tendency. This discrepancy may represent a difference between ongoing tonic sigma activity throughout NREM sleep as opposed to sigma activity specifically linked to the oscillatory action of distinct spindle events. In addition, the focus of the current study was to investigate interrelationships between cognitive abilities and trait-like sleep features in natural, undisturbed sleep including sleep spindles, sleep quality, and indicators of circadian rhythmicity. In line with previous studies (Dang-Vu et al., 2010; Cote et al., 2000), we believe that it would be important to explore the interrelationships between intellectual ability, sleep spindles, and sleep quality in the face of external stimuli in future studies.

In conclusion, the current study supports previous research (for a review, see Fogel \& Smith, 2011) that identified a relationship between sleep spindles and human intellectual ability. More specifically, the results of this study suggest that fast parietal spindles but not slow frontal spindles in both NREM2 and SWS were directly related to reasoning abilities, but not to verbal or STM abilities, and this relationship does not appear to be mediated by possibly confounding and interrelated factors such as sleep quality or circadian rhythmicity. This indicates that sleep spindles and cognitive ability are directly related and not an epiphenomena of sleep quality or circadian factors.

\section{Acknowledgments}

This research was funded by a Canada Excellence Research Chair Grant to A. M. O., with scholarship support to V. S. from an Ontario Graduate Scholarship.
Reprint requests should be sent to Stuart M. Fogel, The Brain \& Mind Institute, Western University, Room 236, Natural Sciences Centre, London, Ontario, Canada N6A 5B7, or via e-mail: sfogel@ uwo.ca.

\section{REFERENCES}

Achilles, G. M. (2003). Individual differences in morningnesseveningness and patterns of psychological functioning, social adaptation and family stress. Dissertation Abstracts International, Section B: The Science and Engineering, 64, 6-B.

Albouy, G., Fogel, S., Pottiez, H., Nguyen, V. A., Ray, L., Lungu, O., et al. (2013). Daytime sleep enhances consolidation of the spatial but not motoric representation of motor sequence memory. PLoS One, 8, e52805.

Baddeley, A. D. (1968). A 3 min reasoning test based on grammatical transformation. Psychonomic Science, 10, 341-342.

Barakat, M., Doyon, J., Debas, K., Vandewalle, G., Morin, A., Poirier, G., et al. (2011). Fast and slow spindle involvement in the consolidation of a new motor sequence. Behavioural Brain Research, 217, 117-121.

Baron, R. M., \& Kenny, D. A. (1986). The moderator-mediator variable distinction in social psychological research: Conceptual, strategic, and statistical considerations. Journal of Personality and Social Psychology, 51, 1173-1182.

Bastien, C. H., Fortier-Brochu, É., Rioux, I., LeBlanc, M., Daley, M., \& Morin, C. M. (2003). Cognitive performance and sleep quality in the elderly suffering from chronic insomnia. Journal of Psychosomatic Research, 54, 39-49.

Beck, A. T., Epstein, N., Brown, G., \& Steer, R. A. (1988). An inventory for measuring clinical anxiety: Psychometric properties. Journal of Consulting and Clinical Psychology, 56, 893-897.

Beck, A. T., Rial, W. Y., \& Rickels, K. (1974). Short form of depression inventory: Cross-validation. Psychological Reports, 34, 1184-1186.

Bixler, E. O., \& Rhodes, J. M. (1968). Spindle activity during sleep in cultural-familial mild retardates. Psychophysiology, 5, 212.

Blackwell, T., Yaffe, K., Ancoli-Israel, S., Schneider, J. L., Cauley, J. A., Hillier, T. A., et al. (2006). Poor sleep is associated with impaired cognitive function in older women: The study of osteoporotic fractures. Journals of Gerontology, Series A, Biological Sciences and Medical Sciences, 61, 405-410.

Bódizs, R., Kis, T., Lázár, A. S., Havrán, L., Rigó, P., Clemens, Z., et al. (2005). Prediction of general mental ability based on neural oscillation measures of sleep. Journal of Sleep Research, 14, 285-292.

Bódizs, R., Lázár, A. S., \& Rigó, P. (2008). Correlation of visuospatial memory ability with right parietal EEG spindling during sleep. Acta Physiologica Hungarica, 95, 297-306.

Bonjean, M., Baker, T., Lemieux, M., Timofeev, I., Sejnowski, T., \& Bazhenov, M. (2011). Corticothalamic feedback controls sleep spindle duration in vivo. Journal of Neuroscience, 31, 9124-9134

Bonnet, M., \& Arand, D. (1995). Consolidated and distributed nap schedules and performance. Journal of Sleep Research, 4, 71-77.

Borbely, A. A. (1982). A two process model of sleep regulation. Human Neurobiology, 1, 195-204.

Boyle, G. J. (1988). Contribution of Cattelan psychometrics to the elucidation of human intellectual structure. Multivariate Experimental Clinical Research, 8, 267-273.

Cattell, R. B. (1949). Culture Free Intelligence Test, scale 1, handbook. Champaign, IL: Institute of Personality and Ability. 
Clemens, Z., Fabó, D., \& Halász, P. (2005). Overnight verbal memory retention correlates with the number of sleep spindles. Neuroscience, 132, 529-535.

Collins, P., Roberts, A. C., Dias, R., Everitt, B. J., \& Robbins, T. W. (1998). Perseveration and strategy in a novel spatial self-ordered sequencing task for nonhuman primates: Effects of excitotoxic lesions and dopamine depletions of the prefrontal cortex. Journal of Cognitive Neuroscience, 10, 332-354.

Corsi, P. (1972). Human memory and the medial temporal region of the brain [PhD thesis]. Montreal, Canada: McGill University.

Cote, K. A., Epps, T. M., \& Campbell, K. (2000). The role of the spindle in human information processing of high-intensity stimuli during sleep. Journal of Sleep Research, 9, 19-26.

Daan, S., Beersma, D. G., \& Borbely, A. A. (1984). Timing of human sleep: Recovery process gated by a circadian pacemaker. American Journal of Physiology, 246, R161-R183.

Dang-Vu, T. T., McKinney, S. M., Buxton, O. M., Solet, J. M., \& Ellenbogen, J. M. (2010). Spontaneous brain rhythms predict sleep stability in the face of noise. Current Biology, 20, R626-R627.

Dang-Vu, T. T., Salimi, A., Boucetta, S., Wenzel, K., O’Byrne, J., Brandewinder, M., et al. (2015). Sleep spindles predict stress-related increases in sleep disturbances. Frontiers in Human Neuroscience, 9, 68.

De Gennaro, L., Ferrara, M., Vecchio, F., Curcio, G., \& Bertini, M. (2005). An electroencephalographic fingerprint of human sleep. Neuroimage, 26, 114-122.

Delorme, A., \& Makeig, S. (2004). EEGLAB: An open source toolbox for analysis of single-trial EEG dynamics including independent component analysis. Journal of Neuroscience Methods, 134, 9-21.

Dijk, D., \& Czeisler, C. (1995). Contribution of the circadian pacemaker and the sleep homeostat to sleep propensity, sleep structure, electroencephalographic slow waves, and sleep spindle activity in humans. Journal of Neuroscience, 15 , 3526-3538.

Dijk, D. J., \& Cajochen, C. (1997). Melatonin and the circadian regulation of sleep initiation, consolidation, structure, and the sleep EEG. Journal of Biological Rhythms, 12, 627-635.

Dijk, D. J., Hayes, B., \& Czeisler, C. A. (1993). Dynamics of electroencephalographic sleep spindles and slow wave activity in men: Effect of sleep deprivation. Brain Research, 626, 190-199.

Dijk, D. J., Roth, C., Landolt, H., Werth, E., Aeppli, M., Achermann, P., et al. (1995). Melatonin effect on daytime sleep in men: Suppression of EEG low frequency activity and enhancement of spindle frequency activity. Neuroscience Letters, 201, 13-16.

Douglass, A. B., Bornstein, R. F., Nino-Murcia, G., Keenan, S., Miles, L., Zarcone, V. P., Jr., et al. (1994). The Sleep Disorders Questionnaire: I. Creation and multivariate structure of SDQ. Sleep, 17, 160.

Elton, M., Winter, O., Heslenfeld, D., Loewy, D., Campbell, K., \& Kok, A. (1997). Event-related potentials to tones in the absence and presence of sleep spindles. Journal of Sleep Research, 6, 78-83.

Fogel, S., Albouy, G., King, B., Vien, C., Karni, A., Benali, H., et al. (2014). Motor memory consolidation depends upon reactivation driven by the action of sleep spindles. Journal of Sleep Research, 23(Suppl. 1), 47.

Fogel, S., \& Jacob, J. S. (2001). Increased sleep spindle activity following simple motor procedural learning in humans. Proceedings of the Congress Physiological Basis for Sleep Medicine, 7, 123.

Fogel, S., Nader, R. S., Cote, K. A., \& Smith, C. (2007). Sleep spindles and learning potential. Behavioral Neuroscience, 121, 1-10.
Fogel, S., Ray, L. B., Binnie, L., \& Owen, A. M. (2015). How to become an expert: A new perspective on the role of sleep in the mastery of procedural skills. Neurobiology of Learning and Memory, 125, 236-248.

Fogel, S., \& Smith, C. (2011). The function of the sleep spindle: A physiological index of intelligence and a mechanism for sleep-dependent memory consolidation. Neuroscience $\mathcal{E}$ Biobehavioral Reviews, 35, 1154-1165.

Fogel, S., \& Smith, C. T. (2006). Learning-dependent changes in sleep spindles and stage 2 sleep. Journal of Sleep Research, 15, 250-255.

Fogel, S. M., \& Jacob, J. S. (2002). The role of sleep spindles in simple motor procedural learning. Sleep, 25, A279-A280.

Folstein, M. F., Folstein, S. E., \& McHugh, P. R. (1975). "Minimental state." A practical method for grading the cognitive state of patients for the clinician. Journal of Psychiatric Research, 12, 189-198.

Gaillard, J. M., \& Blois, R. (1981). Spindle density in sleep of normal subjects. Sleep, 4, 385.

Gais, S., Mölle, M., Helms, K., \& Born, J. (2002). Learningdependent increases in sleep spindle density. Journal of Neuroscience, 22, 6830-6834.

Gibbs, E. L., \& Gibbs, F. A. (1962). Extreme spindles: Correlation of electroencephalographic sleep pattern with mental retardation. Science, 138, 1106-1107.

Gould, R. L., Brown, R. G., Owen, A. M., Bullmore, E. T., \& Howard, R. J. (2006). Task-induced deactivations during successful paired associates learning: An effect of age but not Alzheimer's disease. Neuroimage, 31, 818-831.

Hampshire, A., Highfield, R. R., Parkin, B. L., \& Owen, A. M. (2012). Fractionating human intelligence. Neuron, 76, 1225-1237.

Hauri, P. J. (1997). Cognitive deficits in insomnia patients: The relationship between sleep and cognitive functions during wakefulness. Acta Neurologica Belgica, 97, 113-117.

Hidalgo, M. P. L., Zanette, C. B., Pedrotti, M., Souza, C. M., Nunes, P. V., \& Chaves, M. L. F. (2004). Performance of chronotypes on memory tests during the morning and the evening shifts. Psychological Reports, 95, 75-85.

Horne, J. A., \& Ostberg, O. (1976). A self-assessment questionnaire to determine morningness-eveningness in human circadian rhythms. International Journal of Chronobiology, 4, 97-110.

Iber, C., Ancoli-Israel, S., Chesson, A. L., \& Quan, S. F. (2007). The AASM manual for the scoring of sleep and associated events: Rules, terminology and technical specifications. Westchester, IL: American Academy of Sleep Medicine.

Inoue, S., \& Matsuzawa, T. (2007). Working memory of numerals in chimpanzees. Current Biology, 17, R1004-R1005.

Jackson, D. (1998). Multidimensional Aptitude Battery-II manual. London, Canada: Research Psychologists Press, Inc.

Jobert, M., Poiseau, E., Jahnig, P., Schulz, H., \& Kubicki, S. (1992). Topographical analysis of sleep spindle activity. Neuropsychobiology, 26, 210-217.

Lafortune, M., Gagnon, J. F., Martin, N., Latreille, V., Dubé, J., Bouchard, M., et al. (2014). Sleep spindles and rapid eye movement sleep as predictors of next morning cognitive performance in healthy middle-aged and older participants. Journal of Sleep Research, 23, 159-167.

Mongrain, V., Carrier, J., \& Dumont, M. (2005). Chronotype and sex effects on sleep architecture and quantitative sleep EEG in healthy young adults. Sleep, 28, 819-817.

Morin, A., Doyon, J., Dostie, V., Barakat, M., Hadj Tahar, A., Korman, M., et al. (2008). Motor sequence learning increases sleep spindles and fast frequencies in post-training sleep. Sleep, 31, 1149-1156.

Nader, R. S., \& Smith, C. (2001). The relationship between stage 2 sleep spindles and intelligence. Sleep, 24, A160. 
Nader, R. S., \& Smith, C. (2003). A role for stage 2 sleep in memory processing. Sleep and Brain Plasticity, 1, 87-99.

Nebes, R. D., Buysse, D. J., Halligan, E. M., Houck, P. R., \& Monk, T. H. (2009). Self-reported sleep quality predicts poor cognitive performance in healthy older adults. The Journals of Gerontology, Series B, Psychological Sciences and Social Sciences, 64, 180-187.

Peters, A., \& Jones, E. G. (Eds.) (1991). Normal and altered states of function (Vol. 9). Boston: Springer.

Preckel, F., Lipnevich, A. A., Schneider, S., \& Roberts, R. D. (2011). Chronotype, cognitive abilities, and academic achievement: A meta-analytic investigation. Learning and Individual Differences, 21, 483-492.

Rasch, B., \& Born, J. (2013). About Sleep's Role in Memory. Physiological Reviews, 33, 681-766.

Raven, J. C., Court, J. H., \& Raven, J. (1976). Manual for Raven's progressive matrices. London: $\mathrm{H}$. K. Lewis.

Ray, L. B., Sockeel, S., Soon, M., Bore, A., Myhr, A., Stojanoski, B., et al. (2015). Expert and crowd-sourced validation of an individualized sleep spindle detection method employing complex demodulation and individualized normalization. Frontiers in Human Neuroscience, 9, 507.

Roberts, R. D., \& Kyllonen, P. C. (1999). Morningness-eveningness and intelligence: Early to bed, early to rise will likely make you anything but wise! Personality and Individual Differences, 27, 1123-1133.

Schabus, M., Dang-Vu, T. T., Albouy, G., Balteau, E., Boly, M., Carrier, J., et al. (2007). Hemodynamic cerebral correlates of sleep spindles during human non-rapid eye movement sleep. Proceedings of the National Academy of Sciences, U.S.A., 104, 13164-13169.

Schabus, M., Dang-Vu, T. T., Heib, D. P. J., Boly, M., Desseilles, M., Vandewalle, G., et al. (2012). The fate of incoming stimuli during NREM sleep is determined by spindles and the phase of the slow oscillation. Frontiers in Neurology, 3, 40.

Schabus, M., Gruber, G., Parapatics, S., Sauter, C., Klösch, G., Anderer, P., et al. (2004). Sleep spindles and their significance for declarative memory consolidation. Sleep, 27, 1479-1485.

Schabus, M., Hödlmoser, K., Gruber, G., Sauter, C., Anderer, P., Klösch, G., et al. (2006). Sleep spindle-related activity in the human EEG and its relation to general cognitive and learning abilities. European Journal of Neuroscience, 23, 1738-1746.

Schmidt, C., Collette, F., Cajochen, C., \& Peigneux, P. (2007). A time to think: Circadian rhythms in human cognition. Cognitive Neuropsychology, 24, 755-789.

Schmidt, C., Peigneux, P., \& Cajochen, C. (2012). Age-related changes in sleep and circadian rhythms: Impact on cognitive performance and underlying neuroanatomical networks. Frontiers in Neurology, 3, 118.

Shallice, T. (1982). Specific impairments of planning. Philosophical Transactions of the Royal Society, Series B, Biological Sciences, 298, 199-209.

Silverman, I., Choi, J., Mackewn, A., Fisher, M., Moro, J., \& Olshansky, E. (2000). Evolved mechanisms underlying wayfinding. Evolution and Human Behavior, 21, 201-213.

Silverstein, L. D., \& Levy, C. M. (1976). The stability of the sigma sleep spindle. Electroencephalography and Clinical Neurophysiology, 40, 666-670.
Song, J., \& Stough, C. (2000). The relationship between morningness-eveningness, time-of-day, speed of information processing, and intelligence. Personality and Individual Differences, 29, 1179-1190.

Steriade, M. (1991). Alertness, quiet sleep, dreaming. In A. Peters \& E. J. Jones (Eds.), Cerebral cortex (pp. 279-357). New York: Plenum.

Steriade, M. (2005). Sleep, epilepsy and thalamic reticular inhibitory neurons. Trends in Neurosciences, 28, 317-324.

Steriade, M., Nunez, A., \& Amzica, F. (1993). A novel slow $(<1 \mathrm{~Hz})$ oscillation of neocortical neurons in vivo: Depolarizing and hyperpolarizing components. Journal of Neuroscience, 13, 3252.

Stroop, J. R. (1935). Studies of interference in serial verbal reactions. Journal of Experimental Psychology, 18, 643-662.

Taillard, J., Philip, P., Coste, O., Sagaspe, P., \& Bioulac, B. (2003). The circadian and homeostatic modulation of sleep pressure during wakefulness differs between morning and evening chronotypes. Journal of Sleep Research, 12, 275-282.

Treisman, A. M., \& Gelade, G. (1980). A feature-integration theory of attention. Cognitive Psychology, 12, 97-136.

Ujma, P. P., Konrad, B. N., Genzel, L., Bleifuss, A., Simor, P., Pótári, A., et al. (2014). Sleep spindles and intelligence: Evidence for a sexual dimorphism. Journal of Neuroscience, 34, 16358-16368.

von Krosigk, M., Bal, T., \& McCormick, D. A. (1993). Cellular mechanisms of a synchronized oscillation in the thalamus. Science, 261, 361-364.

Vyazovskiy, V. V., Achermann, P., Borbely, A. A., \& Tobler, I. (2004). The dynamics of spindles and EEG slow-wave activity in NREM sleep in mice. Archives Italiennes de Biologie, $142,511-523$

Wamsley, E. J., Shinn, A. K., Tucker, M. A., Ono, K. E., McKinley, S. K., Ely, A. V., et al. (2013). The effects of eszopiclone on sleep spindles and memory consolidation in schizophrenia: A randomized placebo-controlled trial Sleep, 36, 1369-1376.

Wamsley, E. J., Tucker, M. A., Shinn, A. K., Ono, K. E., McKinley, S. K., Ely, A. V., et al. (2012). Reduced sleep spindles and spindle coherence in schizophrenia: Mechanisms of impaired memory consolidation? Biological Psychiatry, 71, 154-161.

Wechsler, D. A. (1981). Wechsler Adult Intelligence Scale-Revised. New York: Psychological Corporation.

Wei, H. G., Riel, E., Czeisler, C. A., \& Dijk, D. J. (1999). Attenuated amplitude of circadian and sleep-dependent modulation of electroencephalographic sleep spindle characteristics in elderly human subjects. Neuroscience Letters, 260, 29-32.

Werth, E., Achermann, P., \& Borbely, A. A. (1997). Frontooccipital EEG power gradients in human sleep. Journal of Sleep Research, 6, 102-112.

Werth, E., Achermann, P., Dijk, D. J., \& Borbely, A. A. (1997). Spindle frequency activity in the sleep EEG: Individual differences and topographic distribution. Electroencephalography and Clinical Neurophysiology, 103, 535-542.

Zeitlhofer, J., Gruber, G., Anderer, P., Asenbaum, S., Schimicek, P., \& Saletu, B. (1997). Topographic distribution of sleep spindles in young healthy subjects. Journal of Sleep Research, 6, 149-155. 